\title{
“A COMPARATIVE EVALUATION OF CONVENTIONAL STAINING METHODS AND IMMUNOLOGICAL TECHNIQUES FOR THE DIAGNOSIS OF MALARIA"
}

\author{
Samir Pachpute, Chitra Pai, A.D. Urhekar
}

1. Assistant Professor, Department of Microbiology, MGM Medical College \& Hospital.

2. Professor, Department of Microbiology, MGM Medical College \& Hospital.

3. Professor \& HOD, Department of Microbiology, MGM Medical College \& Hospital.

\section{CORRESPONDING AUTHOR}

Dr. Samir Pachpute

104, Rajesh Residency,

Plot no 26, sector no 36,

Kamothe, Navi Mumbai- 410209,

E-mail: drsamirpachpute@gmail.com,

Ph: 0091959493775500919820175787.

AIMS AND OBJECTIVES: To compare the conventional staining methods (Leishman stain, JSB stain, field's stain) on peripheral blood smear with rapid immunological methods (Falcivax (HRP2Ag+ pLDH) \& SD Bioline (pLDH)) for the diagnosis of malarial parasites and to achieve at a better diagnostic tool for diagnosis of malarial parasites in terms of cost, technical expertise, lab facilities, rapidity, higher sensitivity and specificity. MATERIALS AND METHODS: The present study was conducted in the Microbiology Department of a MGM Medical College \& Hospital , Navi Mumbai from July 2009 to June 2010. 339 patients with clinical features s/o Malaria were included in the study. For all the patients in the study group, $1 \mathrm{ml}$ of blood was collected by venipuncture aseptically in EDTA bulbs. Thick and thin blood films were prepared on 3 slides one each for Leishman, Fields and JSB stain. All the samples were then subjected to antigen detection using Falcivax (Pf \& Pv) and SD Bioline (Pf \& Pv) Kits. Test was performed as per kit instruction manual and interpreted accordingly. RESULTS: A total of 339 cases were studied, 238 males and 101 females with age ranges from 15yrs to 72yrs. Of 339 cases, 138 cases were positive for malarial parasites by gold standard viz: Leishman stained thick smear. 68 cases were infected with P.falciparum, 51 with P.vivax and 19 had mixed infection. Falcivax kit showed higher sensitivity and specificity compared to rest of all the rapid methods for detection of P.falciparum. In diagnosing P.falciparum malaria it showed sensitivity of $100 \%$ in comparison with gold standard even at low parasitemias. In fact, Falcivax kits also detect falciparum infections even when the parasites are sequestered and not detectable by microscopy. In case of detection of P.vivax, performance of SD bioline and Falcivax kits were similar (96\%) and better than conventional staining of thin blood smear examination (92\%) by Leishman, JSB and Field's method when compared with gold standard thick smear examination. CONCLUSION: The logistic, economic and technical factors limit rapid access to microscopic confirmation of malaria in many tropical countries including India. So taking into account of all the factors from our study, we recommend Falcivax HRP2 antigen detection as a tool for diagnosis of malaria in future. Although no test can completely replace the conventional method of peripheral blood smear examination, these newer diagnostic tests can be used as supplement to microscopic examination of peripheral blood smear where the diagnosis cannot be made on microscopy alone. Also, rapid immunochromatography tests can be used in times of urgency 
and in areas where facilities of microscopy are not available especially during night times when services of laboratory and experienced Microscopists are not available.

INTRODUCTION: Malaria is the most important parasitic disease of mankind and known since antiquity. The human disease is a protozoan infection of red blood cells transmitted by the bite of a blood feeding female anopheline mosquito ${ }^{2}$. Prevalence of malaria is around 300 million people worldwide with a global death rate over 1.5 million/year. ${ }^{1,2}$.

Malaria is a serious disease caused by the protozoal parasite Plasmodium, and if left untreated, can be fatal. Only four of the known species of Plasmodium are able to infect humans: P. falciparum, P. vivax, P.ovale, and P. malariae. The symptoms of malaria include high fevers, chills, rigors, and flu-like illness. Because the symptoms are so familiar, they are often misdiagnosed. The increasing incidence of malaria, the need to identify and treat the additional carriers (reservoirs) and to reduce the chances of transmission has given an impetus for development of simple and rapid methods for the diagnosis of malaria. Conventional Leishman or Giemsa stained peripheral blood smear examination remain the gold standard for the diagnosis of malaria in malaria endemic countries. However it has disadvantages such as it is labor-intensive, time consuming and requires expertise. ${ }^{4}$

Numerous rapid techniques like acridine orange(AO) stain, Quantitative Buffy coat (QBC), detection of soluble histidine rich protein II antigen (HRP2 Ag), pLDH and aldolase enzymes in whole blood have been evaluated to diagnose malaria. Malaria presents a diagnostic challenge to laboratories in most countries. This study attempts to review the current methodology and approach to the diagnosis of malaria in a practical and helpful way for the laboratory and for the physician caring for the patient. The urgency and importance of obtaining results quickly from the examination of blood samples from patients with suspected acute malaria render some of the more sensitive methods like PCR, DNA Probe assay for malaria diagnosis impractical for routine laboratory use, although they may be considered reference procedures. 3,4,5 So, in the present study an attempt is made to evaluate and compare maximum number of rapid, feasible and economical tests for diagnosis of malaria with the Leishman stained thick blood smears which is considered as Gold standard. These tests include JSB stain, Field's stain and rapid immunological techniques viz; Falcivax (HRP2Ag+ pLDH) \& SD bioline (pLDH).

Keeping in mind the seriousness of condition and the current availability of diagnostic modalities, the present study was carried out in a tertiary care hospital in Navi Mumbai with the following aims and objectives 1) To study the comparative efficacy of the peripheral smear examination by Leishman stain, JSB stain, field's stain and rapid immunologically techniques viz; Falcivax (HRP2Ag+ pLDH) \& SD Bioline (pLDH) for diagnosis of Malaria. 2) To determine the most optimum diagnostic tool for diagnosis of malarial parasites in terms of cost, technical expertise, lab facilities, rapidity, higher sensitivity and specificity.

MATERIALS AND METHODS:METHODOLOGY: The present study was conducted in the Microbiology Department of a tertiary Care Hospital from July 2009 to June 2010. The study was cleared by Institutional ethics committee. 339 patients were included in the study.

\section{CRITERIA FOR ENROLLING PATIENTS INCLUSION CRITERIA:}

Patients with clinical features s/o Malaria which includes 
- history of fever within the preceding $48 \mathrm{hrs,}$

- chills and rigors, or

- fever at the time of presentation or

- Fever of unknown origin, with or without splenomegaly, systemic complications or CNS manifestations.

\section{EXCLUSION CRITERIA:}

- Those patients treated for malaria in last 2 weeks.

- Those patients with confirmed diagnosis of infections like typhoid, pneumonia, Urinary tract infection, Septicemia, dengue, or leptospirosis were ruled out from the study.

SAMPLE COLLECTION: For all the patients in the study group, $1 \mathrm{ml}$ of blood is collected by venipuncture aseptically in EDTA bulbs. Ethics committee and Research committee of the institution had approved the study

\section{CONVENTIONAL STAINING METHODS:}

SAMPLE PREPARATION (THIN FILM AND THICK FILM): For diagnosis of malaria, thick and thin smears are prepared from blood collected in EDTA. Thick and thin blood films are prepared on 3 slides one each for Leishman, fields and JSB stain. Thick smear helps in rapid diagnosis, even when the parasitaemia is low. Thin smear is preferred for determination of species and morphological details of the parasite. Thin film also provides information regarding erythrocyte morphology, leukocytes, and platelets.

The thick smear was stained with Leishman stain. Thin smears were stained by all the three staining methods, Leishman stain, JSB stain and Field's Stain as per standard staining techniques.

\section{METHODS OF COUNTING MALARIAL PARASITES IN THICK BLOOD FILMS EXAMINATION OF THE THICK FILM}

- At least 100 good fields are screened before a slide can be pronounced negative.

- Determination of parasites/ $\mu$ l of blood is done by enumerating the number of parasites in relation to a standard number of leukocytes/ $\mu \mathrm{l}(8000)$. Initially smear is examined for the presence of parasites and species identification. Tally counter is used one for counting leucocytes and other for parasites. After counting 200 leucocytes number of parasites is recorded. Then a simple mathematical formula multiplying the number of parasites by 8000 then dividing this figure by 200 (no. of leucocytes counted) is used.

Parasites counted $/$ no. of leucocytes $\mathrm{x} 8000=$ parasites $/ \mu \mathrm{l}$ of blood.

In this study, 200 leucocytes are counted, thus the formula:

No. of parasites $\mathrm{x} 40$

EXAMINATION OF THIN FILM : examine for at least 100 fields to determine whether the blood film is positive or negative for malaria.

RAPID IMMUNOLOGICAL TECHNIQUES: All the samples are then subjected to antigen detection using Falcivax (Pf \& Pv) and SD Bioline (Pf \& Pv) Kits. Test was performed as per kit instruction manual and interpreted accordingly. 
SD MALARIA ANTIGEN: TEST EXPLANATION \& PRINCIPLE: The SD Malaria antigen test contains a membrane strip, which is precoated with two polyclonal antibodies as two separate lines across a test strip. One polyclonal antibodies (test line Pf) are specific to lactate dehydrogenase of P. falciparum and the other polyclonal antibodies (test line P.v/ pan) are Pan specific to the lactate dehydrogenase of plasmodium species.

FALCIVAX RAPID TEST FOR MALARIA (DEVICE): Falcivax is a rapid self performing, qualitative, two site sandwich immunoassay utilizing whole blood for the detection of $P$. falciparum specific histidine rich protein - 2 (HRP-2) and P. vivax specific pLDH. The test can also be used for specific detection and differentiation of P. falciparum and P. vivax malaria in areas with high rate of mixed infections.

TEST PROCEDURE - Were performed as per kit literature All the results of conventional staining methods and rapid immunological techniques are compared and evaluated for the sensitivity and specificity for the diagnosis of malaria.

RESULTS AND OBSERVATIONS: Of the 339 patients, 68 (20\%) were positive for falciparum malaria by Leishman stained thick blood smear, 6017\%) by Leishman stained thin blood smear, $60(17 \%)$ by JSB stained thin smear, 60 (17\%) by Field's stained thin smear, 69(20.3\%) by Falcivax antigen Kit and $57(16.8 \%)$ by SD Bioline antigen Kit. Thin smear stained by Leishman, JSB and Fields gave similar results. They failed to detect $8(11.8 \%)$ of the positives by Gold standard, while SD Bioline method failed to detect 11(16\%) cases. Falcivax detected all the positive cases, but also detected one positive that was negative by Leishman stained Thick smear (gold Standard). All those positives by other methods were also positive by Gold standard.

Of the 339 patients, 51(15\%) were positive for vivax malaria by Gold Standard Leishman stained thick blood smear, $47(13.8 \%)$ by Leishman stained thin blood smear, $47(13.8 \%)$ by JSB stained thin smear, $47(13.8 \%)$ by Field's stained thin smear, $49(14.4 \%)$ by Falcivax antigen Kit and 49(14.4\%) by SD Bioline antigen Kit. thin smear stained by Leishman, JSB and Fields gave similar results. They failed to detect $4(7 \%)$ of the positives by Gold standard, while SD Bioline and Falcivax methods failed to detect 2(3.9\%) cases. All those positives detected by other methods were also positive by Gold standard.

There were 19 positive cases for mixed malarial parasite infection. All the methods under study gave same results for mixed infection as compared to gold standard.

Parasitic index was calculated for all the positive malaria cases by gold standard Leishman stained thick smear. The parasitemias ranged from 80-9600 parasites/ $\mu$ l. Sensitivity of all the rapid conventional staining methods was $100 \%$ at parasitemias $>200$ parasites $/ \mu$ for P.falciparum and 240 parasites/ $\mu$ l for P.vivax. But sensitivity was lowered to $88 \%$ and $92 \%$ for P.falciparum and P.vivax respectively with parasitemias <200parasites/ $\mu$ l. SD Bioline Antigen detection method had a detection threshold of 280parasites/ $\mu$ l for P.vivax and 200parasites/ $\mu$ l. Sensitivity of Falcivax Kit for P.falciparum was $100 \%$ at 80 parasites/ $\mu$. The detection limit for P.vivax was 200 parasites/ $\mu$ l. similar to that of SD Bioline, as for P.vivax, method of detection of vivax specific $\mathrm{pLDH}$ is same in both kits. 
DATA ANALYSIS: Sensitivity, specificity, positive predictive value, negative predictive value were calculated for each method by comparing the proportion of positive and negative results for each method with the gold standard Leishman stained thick blood smear examination.

Prevalence $=$ No. of positives by Leishman stained thick blood smear examination $/$ total no. of samples examined.

Sensitivity $=$ No of matching Positives by both Leishman stained thick smear examination(Gold std) and the method to be evaluated/no. of positives by Leishman stained thick blood smear examination. OR TP/TP+FN. This is no . of True Positives

Specificity $=$ No. of matching negatives by both Leishman stained thick smear examination(Gold std) and the method to be evaluated/no. of positives by Leishman stained thick blood smear examination. OR TN/TN=FN. This is no. of True negatives.

Positive Predictive value (PPV) = sensitivity $\times(1-$ Prevalence $) /$ (sensitivity $x$ prevalence) + (1specificity) $x$ (1- prevalence) OR TP/ TP + FP

Negative predictive value $(\mathrm{NPV})=$ specificity $\times(1-$ Prevalence $) /$ specificity $\times(1$ - prevalence $)+$ (1- sensitivity) x prevalence OR TN/FN+TN

The concordance of tests was calculated as follows ;

Concordance $=$ No. of matching Positives and negatives by both Leishman stained thick smear examination(Gold std) and the method to be evaluated/ total no. of samples examined OR TP+ TN/ Total no. of samples examined.

The values obtained are multiplied by 100 and reported as percentage.

Where, TP-True positive, TN - True negative

With reference to the data shown in Table 10:

Prevalence of malaria $=\mathbf{4 0} \%$

DISCUSSION: The majority of cases of malaria worldwide are treated on the basis of clinical diagnosis and microscopy. Several studies have shown that the ability to diagnose malaria by blood film examination alone is about $75 \%$ for P.falciparum. ${ }^{6}$ Rapid detection and effective treatment of malaria is a prerequisite in reducing the morbidity and mortality due to the disease. Leishman stained thick smear examination, which is the corner stone in the laboratory diagnosis of malaria, has undergone little improvement since its inception. In the present study, We included 339 patients attending hospital over a period of one year for different complaints suggestive of malaria. We evaluated and compared five different techniques of rapid detection of malarial parasites with the Gold standard Leishman stained thick smear.

TYPE OF MALARIA: In India 40\% of cases are as result of P.vivax malarial infection. $44.3 \%$ are due to P.falciparum malarial infection. $10-15 \%$ is due to mixed malarial infection. ${ }^{1}$ In this study, P.falciparum contributed to $49 \%$ of the total study group, P.vivax contributed to $37 \%$ of the total study group and mixed malarial infection was responsible for the remaining of $14 \%$ of the study group.

Mendiratta et al $^{6}$ studied 443 cases with maximum number of P.falciparum cases. Thus our study compares well with WHO report and other similar studies regarding the increased incidence of P.falciparum.

In the present study, while comparing the available different methods of rapid detection for P.falciparum with gold standard Leishman stained thick blood smear microscopy, the sensitivity of Falcivax antigen kit was highest (100\%) followed by Leishman stained thin smear (88\%), JSB stain (88\%), Fields stain (88\%), and lastly by SD Bioline (83\%). For detection of P. 
vivax, Both antigen Kits Falcivax and SD bioline (96\%) detected highest no. of cases followed by thin peripheral smear microscopy by Leishman, JSB and Field's stain (92\%). our results were in agreement with other studies.5,6,7 In our study there were no false positive results by any of the methods suggesting $100 \%$ specificity, except Falcivax kit which showed one false positive for falciparum.

In the present study the results obtained by thin smear examination by Leishman stain, JSB stain and Fields stain were same. They had lower sensitivity of $88 \%$ for P.falciparum and 92 $\%$ for P.vivax. It was found that at lower parasitemia, the sensitivity dropped considerably. Sensitivity was $100 \%$ at Parasitemia level 240 parasites/ $\mu$ l or more. Thick smear provides enhanced sensitivity of blood film technique and is much better than the thin film for detection of low levels of parasitemia and relapse or recrudescence 9 . Warhurst and Williams reported that examination of thin blood films is only $1 / 10$ as sensitive as examination of thick blood films for the quantification of malarial parasites, although morphological identification of the Plasmodium species present is much easier using thin films. It is highly recommended that both thick and thin films be prepared and examined each time blood film examination for parasites is requested. 8

Sensitivity of SD Bioline antigen kit, based on pLDH for P.falciparum was found to be lowest. The recommended method and current gold standard used for the routine laboratory diagnosis of malaria is the microscopic examination of stained thick blood films. In the most capable hands, this method can be expected to detect 50 parasites $/ \mu \mathrm{l}(0.001 \%$ parasitemia $)$ and to identify to the species level $98 \%$ of all parasites seen. In our study parasitemia level of 80 parasites/ $\mu \mathrm{l}$ or more were detected by thick smear microscopy. This procedure is recognized as difficult and time-consuming, requiring considerable training to obtain the necessary skills.

Immunochromatographic dipsticks offer the possibility of more rapid, nonmicroscopic methods for malaria diagnosis, thereby saving on training and time. These tests are easy to perform and require little training to interpret the results. On June 13, 2007, the U.S. Food and Drug Administration (FDA) approved the first RDT for use in the United States. This RDT is approved for use by hospital and commercial laboratories, not by individual clinicians or by patients themselves. It is recommended that all RDTs are followed-up with microscopy to confirm the results and if positive, to quantify the proportion of red blood cells that are infected. ${ }^{10}$

RDT currently on the market are easy to use; most are in cassette format with singleapplication areas for the blood sample and clearing buffer. We have also used Falcivax Kit which is in cassette format which is easier to perform as compared to SD bioline dipstick method. Most evaluation trials have included temperature and time stability for at least 1 year at $40^{\circ} \mathrm{C}$.

The new generation of RDT offers a realistic practical chance to move the diagnosis of malaria away from the laboratory and nearer to the patient. our results with Falcivax Kit which is HRP2 based showed $100 \%$ sensitivity even at 80 parasites/ $\mu$ as compared to pLDH based SD Bioline Kit and peripheral blood smear microscopy for diagnosis of P. falciparum. The added assurance that life-threatening parasitemia with P. falciparum will not be missed is welcome, particularly for inexperienced laboratory staff during night calls. The ability to detect the majority of the non-falciparum malaria cases also makes these tests ideally suited as major backup procedures for malaria diagnosis.

There are many considerations to be taken into account when reviewing the methods for laboratory diagnosis of malaria (Table 6 ), not the least of which are the important factors of availability and cost. The present debate on the introduction of tests based on new technology is welcomed. However, it does not avoid the necessity of reviewing correctly stained thick and thin 
blood films as the standard operating procedure when malaria is suspected or of replacing a current training program for the identification of the Plasmodium species and for detection of parasitemia below the present threshold of detection by RDT.

CONCLUSION: Falcivax kit showed higher sensitivity and specificity compared to rest of all the rapid methods for detection of P.falciparum.

The logistic, economic and technical factors limit rapid access to microscopic confirmation of malaria in many tropical countries including India. So taking into account of all the factors from our study (Table 6), we consider HRP2 antigen detection kit as a tool for diagnosis of malaria in future. Although no test can replace the conventional method of peripheral blood smear examination, these newer diagnostic tests can be used as supplement to microscopic examination of peripheral blood smear where the diagnosis cannot be made on microscopy alone. These tests can be used in times of urgency and in areas where facilities of microscopy are not available especially during night times when services of laboratory and experienced microscopists are not available.

\section{REFERENCES}

1. WHO SE Asia. Implementation of collaborative activities on Roll back Malaria in SE Asia region. June 2000 New Delhi 1-21.

2. Snow R W, Guerra C A, Noor AM, Mvint H Y SI. The global distribution of clinical episodes of P.falciparum malaria. Nature 2005;434:214-7.

3. Shujatullah Fatima, Abida Malik, Haris khan \& Ashraf Malik, Comparison of different diagnostic techniques in plasmodium falciparum cerebral malaria., jvect borne Dis 43, 2006: 186-190.

4. Mendiratta D K, ,Evaluation of different methods for diagnosis of P.falciparum malaria., DK Mendiratta, R. Narang; Indian journal of Medical Microbiology; (2006) 24 (1): 49-51.

5. Parija S C, A Comparative study of blood smear,QBC and antigen detection for diagnosis of malaria;Indian Journal of Pathology and microbiology; 2009;52(2):200202.

6. White NJ Manson's tropical diseases. Ed. Gordon cook, 20 th Edition 1996; Malaria 1087- 1164.

7. Chakraborthy P, Textbook of Medical Parasitology,New Central agency, Plasmodium \& Babesia2004; 84-103.

8. Warhurst, D. C., and J. E. Williams. 1996. Laboratory diagnosis of Malaria. J. Clin. Pathol. 49:533-538.

9. Hunt-Cooke, A. H., P. L. Chiodini, T. Docherty, A. H. Moody, J. Ries, and M. Pinder. 1999. Comparison of a parasite lactate dehydrogenase-based immunochromatographic antigen detection assay (OptiMAL®) with microscopy for the detection of malaria parasites in human blood samples. Am. J. Trop. Med. Hyg. 60:20-23.

10. Craig, M., and B. L. Sharp. 1997. Comparative evaluation of four techniques for the diagnosis of Plasmodium infections. Trans. R. Soc. Trop. Med. Hyg. 91:279-282.

11. http://www.cdc.gov/malaria/features/index_20041220.htm 
TABLE 1 : Sex distribution of clinically suspected patients of malaria $(n=339)$

\begin{tabular}{|l|l|l|c|}
\hline & $\begin{array}{l}\text { MALES } \\
\text { No. (\%) }\end{array}$ & $\begin{array}{l}\text { FEMALES } \\
\text { No. (\%) }\end{array}$ & TOTAL \\
\hline $\begin{array}{l}\text { Clinically } \\
\text { suspected } \\
\text { patients }\end{array}$ & $238(70 \%)$ & $101(30 \%)$ & 339 \\
\hline
\end{tabular}

TABLE 2: Age group Leishman stained thick blood smear positive cases for malaria

\begin{tabular}{|c|c|c|c|c|c|c|}
\hline & & & P.falciparum & P.vivax & Mix & Total \\
\hline \multirow[t]{3}{*}{$\begin{array}{l}\text { Age } \\
\text { group }\end{array}$} & $\begin{array}{l}15- \\
25 \\
\text { yrs }\end{array}$ & $\begin{array}{l}\text { Count } \\
\text { \% within } \\
\text { age group }\end{array}$ & $\begin{array}{r}11 \\
37.90 \%\end{array}$ & $\begin{array}{r}13 \\
44.80 \%\end{array}$ & $\begin{array}{r}5 \\
17.20 \%\end{array}$ & $100.00 \%$ \\
\hline & $\begin{array}{l}25- \\
50 \\
\text { yrs }\end{array}$ & $\begin{array}{l}\text { Count } \\
\text { \% within } \\
\text { age group }\end{array}$ & $\begin{array}{r}53 \\
53.5 \%\end{array}$ & $\begin{array}{r}31 \\
31.2 \%\end{array}$ & $\begin{array}{r}14 \\
14.3 \%\end{array}$ & $100.00 \%$ \\
\hline & $\begin{array}{l}50- \\
75 \\
\text { yrs }\end{array}$ & $\begin{array}{l}\text { Count } \\
\% \text { within } \\
\text { age group }\end{array}$ & $36.40 \%$ & $63.60 \%$ & $0.00 \%$ & $100.00 \%$ \\
\hline Total & & $\begin{array}{l}\text { Count } \\
\% \text { within } \\
\text { age group }\end{array}$ & $\begin{array}{r}68 \\
49.2 \%\end{array}$ & $\begin{array}{r}51 \\
37 \%\end{array}$ & $\begin{array}{r}19 \\
13.8 \%\end{array}$ & $\begin{array}{r}138 \\
100.00 \%\end{array}$ \\
\hline
\end{tabular}

TABLE 3: Comparison of conventional Leishman stained thick smear examination with other rapid staining methods and rapid immunological techniques for the diagnosis of malarial parasites

\begin{tabular}{|c|c|c|c|c|c|c|}
\hline & Leishman( gold std) & Leishman stain & JSB stain & $\begin{array}{l}\text { Field's } \\
\text { stain }\end{array}$ & $\begin{array}{l}\text { Falcivax } \\
\text { kit }\end{array}$ & $\begin{array}{l}\text { SD } \\
\text { bioline }\end{array}$ \\
\hline & Thick Smear & Thin Smear & $\begin{array}{l}\text { Thin } \\
\text { Smear }\end{array}$ & $\begin{array}{l}\text { Thin } \\
\text { Smear }\end{array}$ & & \\
\hline Negative & 201 & 213 & 213 & 213 & 202 & 214 \\
\hline $\mathrm{PF}$ & 68 & 60 & 60 & 60 & 69 & 57 \\
\hline PV & 51 & 47 & 47 & 47 & 49 & 49 \\
\hline MIX & 19 & 19 & 19 & 19 & 19 & 19 \\
\hline Total & 339 & 339 & 339 & 339 & 339 & 339 \\
\hline
\end{tabular}


TABLE 4: Comparison of sensitivity and specificity of various rapid methods for P.falciparum with Leishman stained thick smear.

\begin{tabular}{|l|l|l|l|l|l|}
\hline & Leishman & JSB & Field's & Falcivax & SD bioline \\
\hline & Thin smear & Thin smear & Thin smear & & \\
\hline Sensitivity & $88.2 \%$ & $88.2 \%$ & $88.2 \%$ & $100 \%$ & $83.8 \%$ \\
\hline Specificity & $100 \%$ & $100 \%$ & $100 \%$ & $99.6 \%$ & $100 \%$ \\
\hline PPV & $100 \%$ & $100 \%$ & $100 \%$ & $98.5 \%$ & $100 \%$ \\
\hline NPV & $97 \%$ & $97 \%$ & $97 \%$ & $100 \%$ & 96.2 \\
\hline $\begin{array}{l}\text { test efficiency } \\
\begin{array}{l}\text { Concordance of } \\
\text { tests) }\end{array}\end{array}$ & $97.6 \%$ & $97.6 \%$ & $97.6 \%$ & 99.7 & 96.7 \\
\hline
\end{tabular}

TABLE 5: Comparison of sensitivity and specificity of various rapid methods for P.vivax with Leishman stained thick smear.

\begin{tabular}{|l|c|c|c|c|c|}
\hline & Leishman & \multicolumn{1}{|l|}{ JSB } & Field's & Falcivax & SD bioline \\
\hline & Thin Smear & Thin smear & Thin smear & & \\
\hline Sensitivity & $92.0 \%$ & $92.0 \%$ & $92.0 \%$ & $96.0 \%$ & $96.0 \%$ \\
\hline Specificity & $100 \%$ & $100 \%$ & $100 \%$ & $100 \%$ & $100 \%$ \\
\hline PPV & $100 \%$ & $100 \%$ & $100 \%$ & $100 \%$ & $100 \%$ \\
\hline NPV & $98.6 \%$ & $98.6 \%$ & $98.6 \%$ & $99.3 \%$ & 99.3 \\
\hline $\begin{array}{l}\text { Test efficiency } \\
\text { (Concordance of } \\
\text { tests) }\end{array}$ & $98.8 \%$ & $98.8 \%$ & $98.8 \%$ & 99.4 & 99.4 \\
\hline
\end{tabular}

For mixed malarial parasite infection, results by all the methods were same as compared to the gold standard. 
TABLE 6: Comparative evaluation of different methods based on following parameters for diagnosis of malaria.

\begin{tabular}{|c|c|c|c|c|c|c|c|}
\hline & Parameters & $\begin{array}{l}\text { Leishman } \\
\text { thick smear } \\
\text { Stain }\end{array}$ & $\begin{array}{l}\text { Leishman } \\
\text { thin } \\
\text { smear } \\
\text { stain }\end{array}$ & JSB stain & $\begin{array}{l}\text { Field's } \\
\text { Stain }\end{array}$ & Falcivax Kit & SD Bioline kit \\
\hline 1 & Sensitivity & $\begin{array}{l}\text { 80parasites/ } \\
\mu \mathrm{l}\end{array}$ & $\begin{array}{l}\text { 240parasit } \\
\text { es/ } \mu \mathrm{l}\end{array}$ & $\begin{array}{l}\text { 240parasit } \\
\text { es/ } \mu \mathrm{l}\end{array}$ & $\begin{array}{l}\text { 240parasite } \\
\mathrm{s} / \mu \mathrm{l}\end{array}$ & $\begin{array}{l}\text { 80parasites } / \mu \\
1\end{array}$ & $\begin{array}{l}\text { 200parasites } / \mu \\
\text { l }\end{array}$ \\
\hline 2 & Specificity & All species & All species & All species & All species & $\begin{array}{l}\text { P.falciparum } \\
\text { and p.vivax }\end{array}$ & $\begin{array}{l}\text { P.falicparum } \\
\text { and P.vivax }\end{array}$ \\
\hline 3 & Cost & Rs 5/test & Rs5/test & Rs 5/test & Rs 5 /test & Rs. 100/test & Rs 60/ test \\
\hline 4 & $\begin{array}{l}\text { Technical } \\
\text { expertise }\end{array}$ & $\begin{array}{l}\text { Highly } \\
\text { skilled }\end{array}$ & $\begin{array}{l}\text { Highly } \\
\text { skilled }\end{array}$ & $\begin{array}{l}\text { highly } \\
\text { skilled }\end{array}$ & $\begin{array}{l}\text { Highly } \\
\text { skilled }\end{array}$ & $\begin{array}{l}\text { no special } \\
\text { skills }\end{array}$ & minimal skills \\
\hline 5 & Equipment & $\begin{array}{l}\text { lab, } \\
\text { microscope, } \\
\text { electricity }\end{array}$ & $\begin{array}{l}\text { Lab, } \\
\text { microscop } \\
\text { e, } \\
\text { electricity }\end{array}$ & $\begin{array}{l}\text { lab, } \\
\text { microscop } \\
\text { e, } \\
\text { electricity }\end{array}$ & $\begin{array}{l}\text { lab, } \\
\text { microscope, } \\
\text { electricity }\end{array}$ & $\begin{array}{l}\text { Kit only. no } \\
\text { special } \\
\text { equipment }\end{array}$ & $\begin{array}{l}\text { Kit only, No } \\
\text { special } \\
\text { equipment }\end{array}$ \\
\hline 6 & $\begin{array}{l}\text { Time for } \\
\text { result }\end{array}$ & 45-60mins & $30-45 \mathrm{mins}$ & $\begin{array}{l}20-30 \\
\text { mins }\end{array}$ & 20-30 mins & $5-15$ mins & $5-15 \operatorname{mins}$ \\
\hline 7 & $\begin{array}{l}\text { Subjectivity } \\
\text { Variation }\end{array}$ & High & High & High & High & Low & Low \\
\hline
\end{tabular}

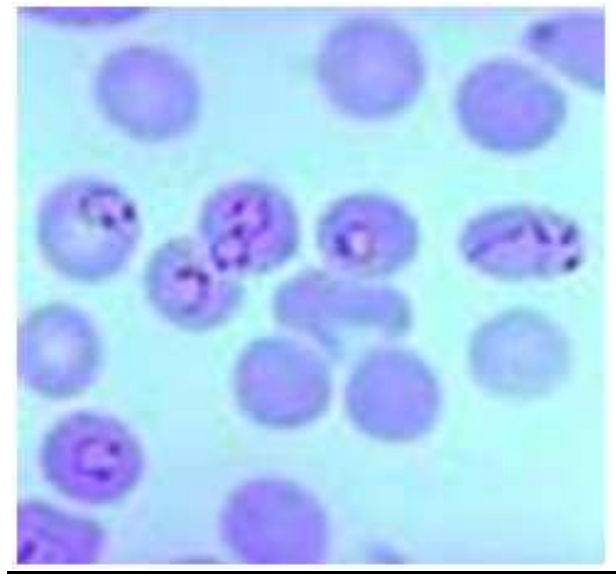

Fig.1 JSB Stained thin smear: P.falciparum rings - multiple rings in one $\mathrm{RBC}$, accole forms

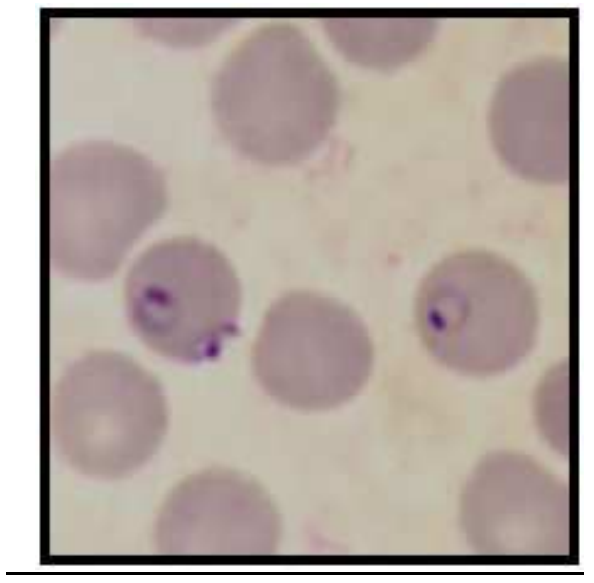

Fig.2 Field's stained thin smear : multiple rings of P.falciparum. 


\section{ORIGINAL ARTICLE}

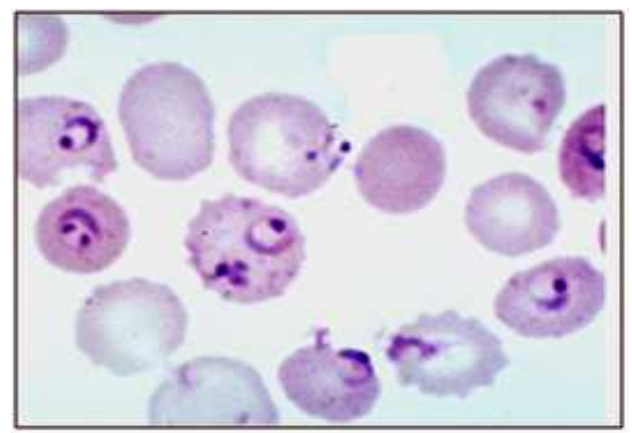

Fig 3. Leishman stained thin smear::

P.falciparum multiple rings, accole forms

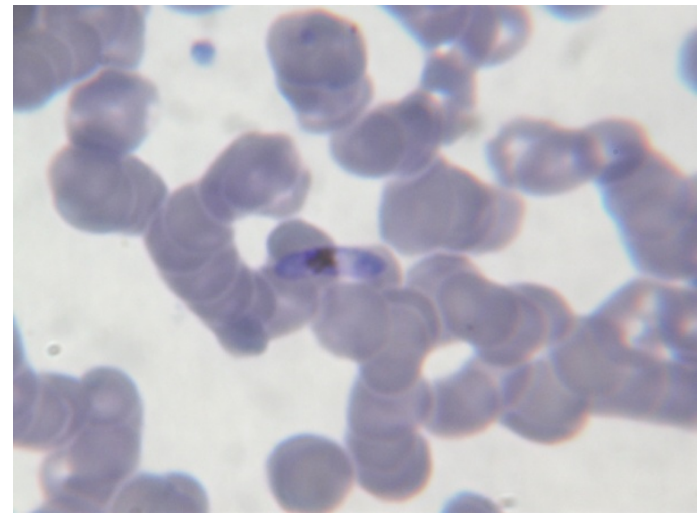

Fig 5. Fields stained thin smear:

P.falciparum gametocyte

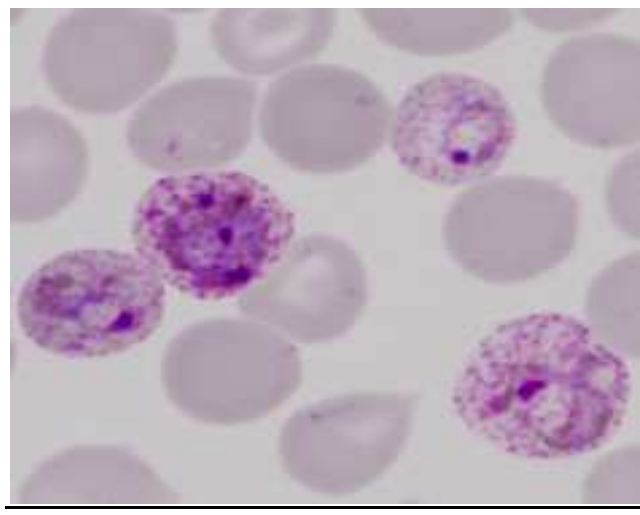

Fig 7. Field's stained thin smear:

P. vivax trophozoites (Ring) with

Schuffner's dots in RBC cytoplasm

\& Schizonts of P.vivax

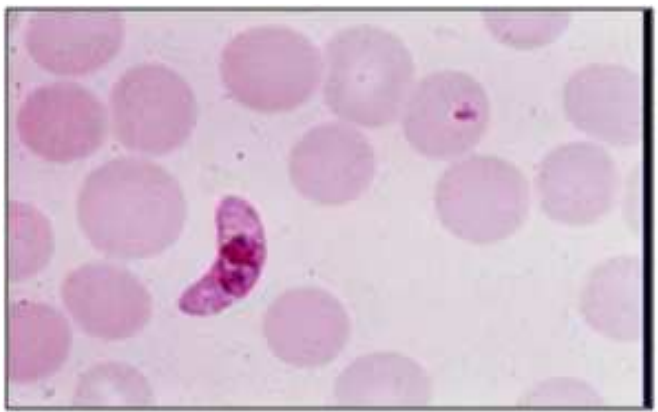

Fig4. JSB stained thin smear:

P.falciparum gametocyte

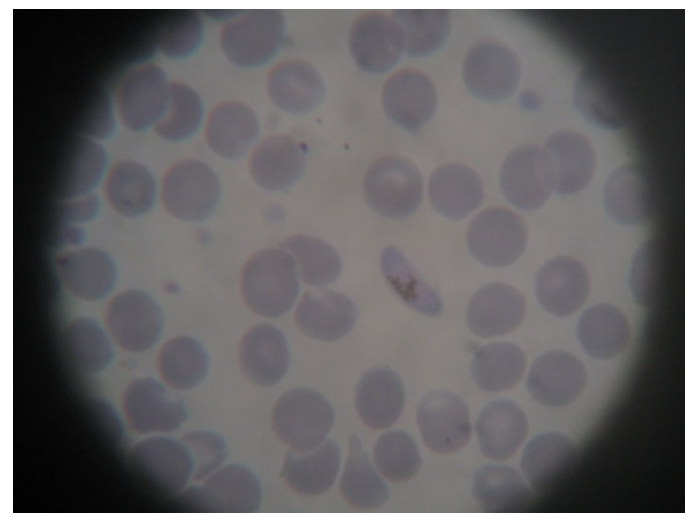

Fig 6. Leishman stained thin smear: P.falciparum gametocyte

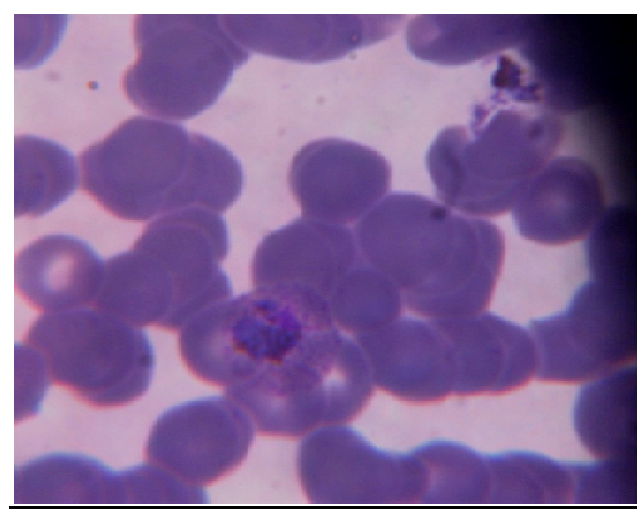

Fig 8. Leishman stained Thin smear:

P. vivax schizont 


\section{ORIGINAL ARTICLE}

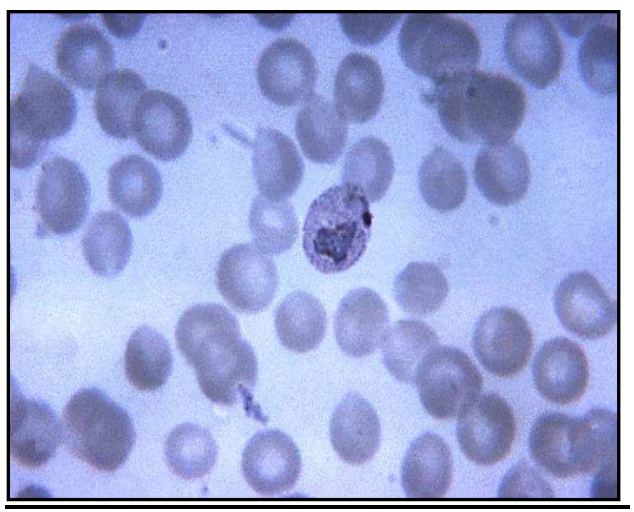

Fig 9. Leishman stain: Schizont of schizont

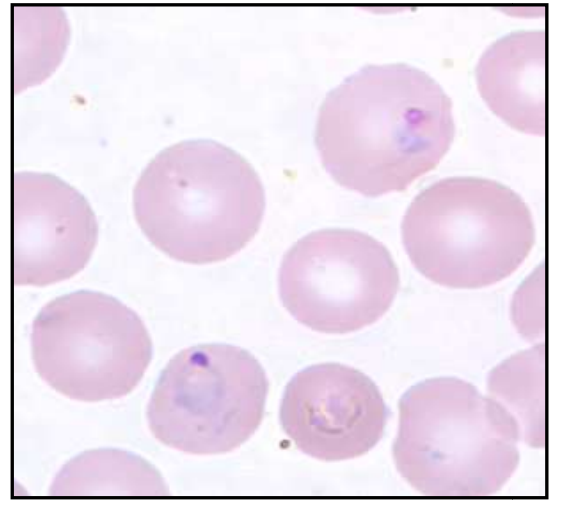

Fig 11. Lesihman's Stain: Ring form of P.vivax

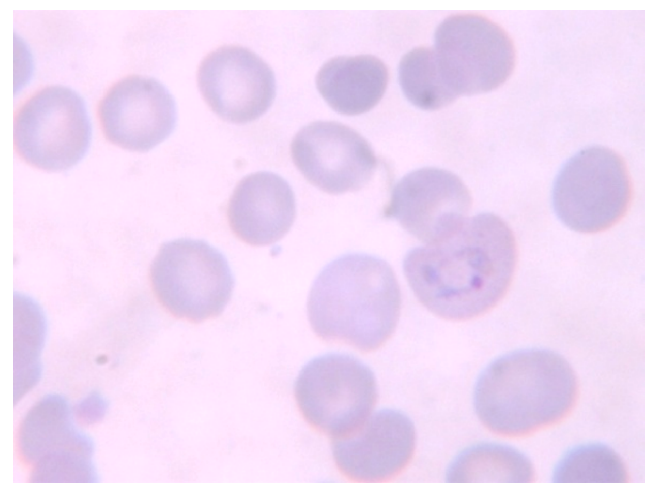

Fig 13. JSB stain : Ring form of P.vivax

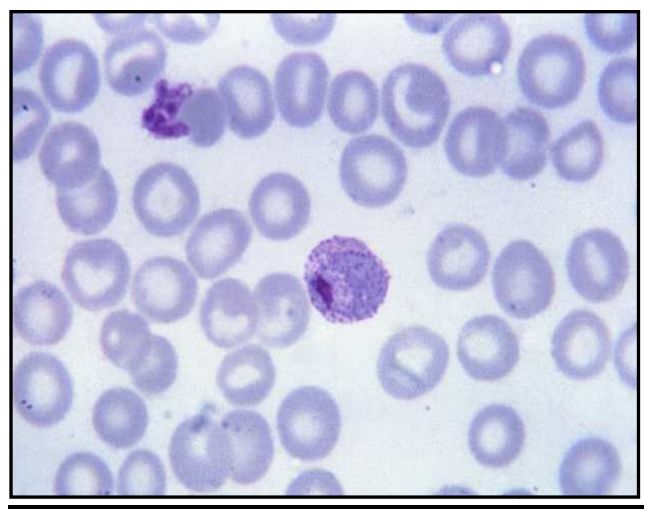

Fig 10. JSB stained thin smear; P.vivax P.vivax

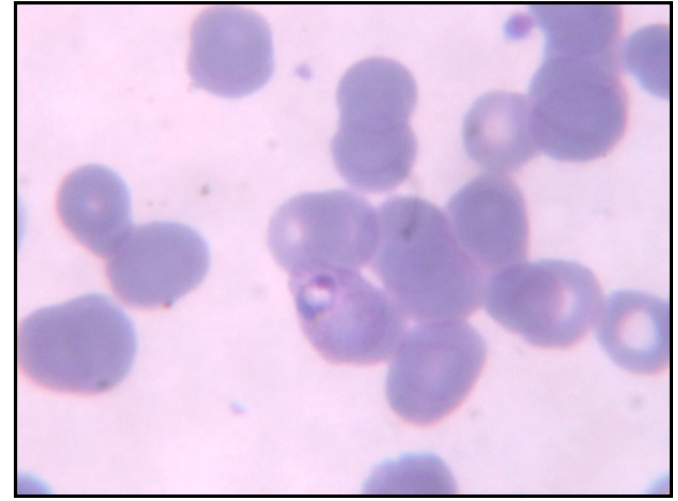

Fig 12. Field's Stain: Ring form of P.vivax

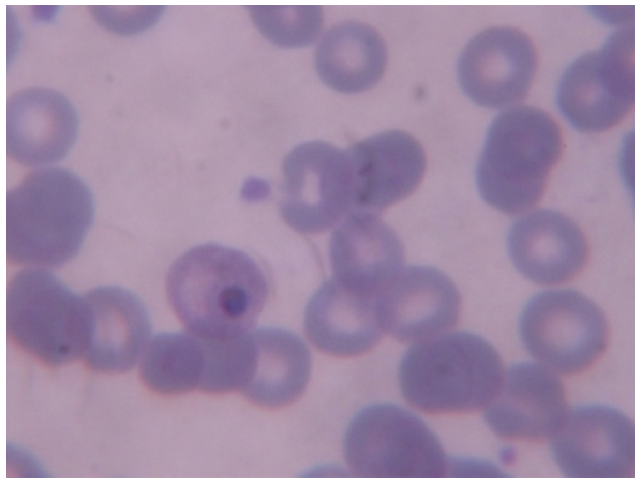

Fig14. JSB Stain: Gametocyte of P.vivax 


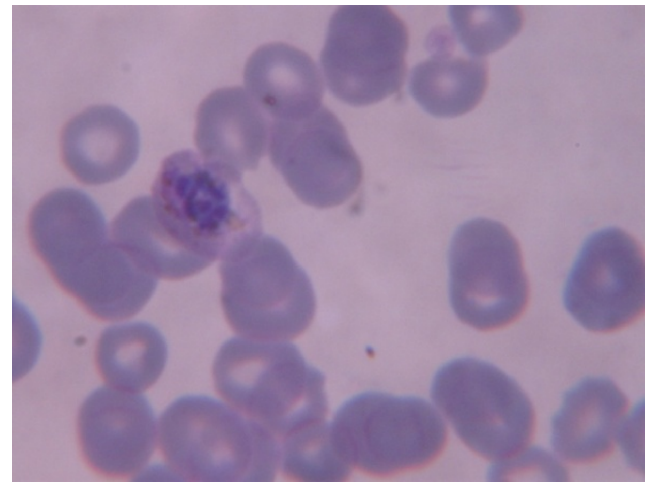

Fig 15. JSB Stain: Schizont with merozoites

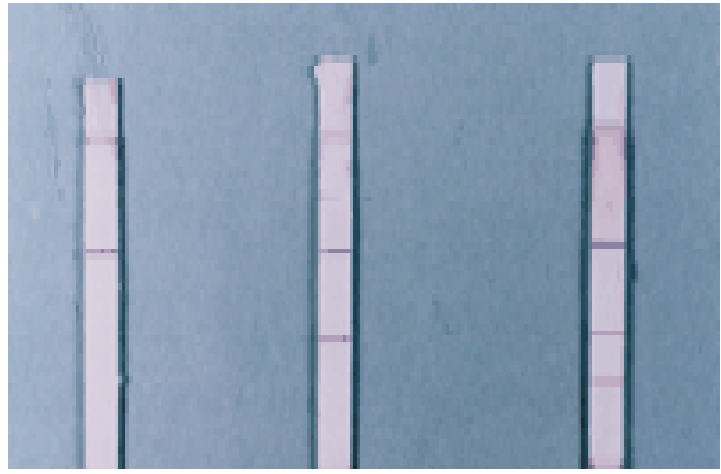

1. Negative 2.P. vivax 3. P.falciparum Fig.16. Interpretation of the test

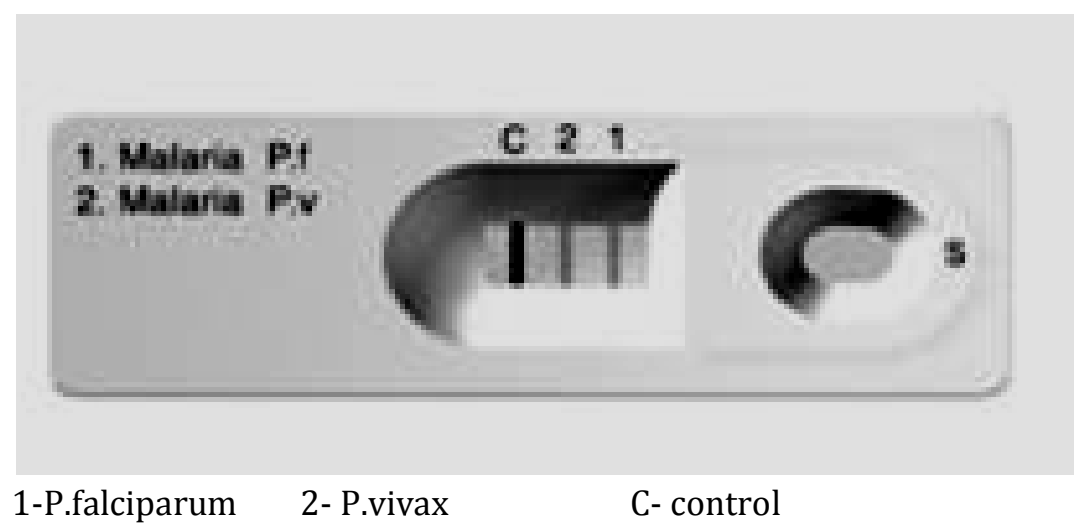

Fig17. Test is positive for P.falciparum and P.vivax 